\title{
Revistas de ciencias agrícolas de Suramérica indexadas en SciELO Relación con indicadores de desarrollo de la agricultura
}

\section{Rene Antonio Hinojosa Benavides}

Universidad Nacional Autónoma de Huanta, Perú | renehinojosa123@yahoo.com / ORCID: https://orcid.org/oooo-0oo2-0452-3162

\author{
Ruggerths Neil de la Cruz Marcos \\ Universidad Nacional de Huancavelica, Perú | https://orcid.org/oooo-ooo2-7539-4003 \\ Carlos Enrique Espinoza Quispe \\ Universidad Nacional de Huancavelica, Perú | https://orcid.org/oooo-0oo3-2843-7426
}

\begin{abstract}
Resumen
En este artículo se analizan indicadores bibliométricos de las revistas indexadas en SciELO pertenecientes al área de ciencias agrícolas para el año 2019 y su relación con indicadores de desarrollo de la agricultura en la región. Los datos bibliométricos fueron obtenidos del portal oficial de SCiELO y los de desarrollo de agricultura se obtuvieron del portal del Banco Mundial. Los datos fueron procesados mediante técnicas estadísticas de asociación por conglomerados para observar similitudes o diferencias entre los indicadores por país. Las relaciones entre los indicadores se establecieron mediante análisis de correlaciones canónicas y árboles de clasificación. El análisis de conglomerados mostró que los países de agrupan según los indicadores bibliométricos en cinco clústeres donde Argentina, Brasil, Chile y Colombia son independientes y Bolivia, Paraguay, Perú y Venezuela se agrupan en un solo clúster. En cuanto a los indicadores de desarrollo de agricultura, se evidencian cuatro clústeres, Bolivia y Paraguay ubicados independientes, Argentina y Venezuela en un clúster y Brasil, Chile, Colombia y Perú en otro. Se evidenció poca relación entre los indicadores bibliométricos y los de desarrollo de agricultura, con predominio de relaciones inversas moderadas con la industrialización y el valor agregado de la agricultura (\%PIB).
\end{abstract}

South American agricultural science journals indexed in SciELO:

Relationship with agricultural development indicators

\footnotetext{
Abstract

This article analyzes bibliometric indicators of the journals indexed in SciELO belonging to the area of agricultural sciences for the year 2019 and their relationship with indicators of agricultural development in the region. The bibliometric data were

Palabras clave

Ciencias agrícolas SciELO

Desarrollo Bibliometría Suramérica

Keywords

Agricultural sciences SCiELO

Development

Bibliometrics

South America 
obtained from the official SciELO website and the agricultural development data were obtained from the World Bank website. The data were processed using statistical techniques of association by clusters to observe similarities or differences between the indicators by country. The relationships between the indicators were established through analysis of canonical correlations and classification trees. The cluster analysis showed that the countries are grouped according to bibliometric indicators in five clusters where Argentina, Brazil, Chile and Colombia are independent and Bolivia, Paraguay, Peru and Venezuela are grouped in a single cluster. As for agricultural development indicators, there are four clusters with Bolivia and Paraguay located independently, Argentina and Venezuela in one cluster and Brazil, Chile, Colombia and Peru in another cluster. There was little relationship between bibliometric indicators and agricultural development indicators, with a predominance of moderate inverse relationships with industrialization and agricultural value added (\%GDP).

Artículo recibido: 29-07-2020. Aceptado: 23-10-2020

\section{Introducción}

Las revistas científicas constituyen el medio de difusión para el conocimiento científico en todas las áreas temáticas. De manera específica, las revistas del área de las ciencias agrícolas tienen por objetivo la divulgación de las investigaciones en el campo de la agronomía y ciencias afines como la agroindustria, la zootecnia, las ciencias forestales, y las ambientales, entre otras (Sangerman-Jarquín, Nuñez y Navarro-Bravo, 2015). La difusión de la ciencia en la actualidad es más evidente e importante que nunca, pues los medios de comunicación digitales han contribuido a su disponibilidad para un amplio público, sobre todo por los sistemas de acceso abierto (Mazzaro, 2010; Sánchez-Tarragó et al., 2016).

En Suramérica se cuenta con diversidad de climas que permiten el desarrollo de la agricultura, además de la cordillera andina, la cual está considerada una de las principales zonas de domesticación vegetal del mundo (Rodríguez, 2010; Salaverry, 2012), sin embargo, la investigación y su difusión está limitada por el bajo número de revistas indexadas en bases de datos de importancia tanto regional como global. La agricultura y la agroindustria se han convertido en un bastión importante en la economía de los países de la región y ha llegado a representar una proporción incluso mayor, en su tamaño relativo, a la economía total de los países (Escobar, 2016), lo que se ha visto favorecido por la siembra de plantas mejoradas genéticamente, el uso de fertilizantes químicos de origen mineral, mejores agroquímicos para el control de plagas, maquinaria agrícola para todas las labores, mejoramiento en los sistemas de riego y otras tecnologías (Gliessman, 2013; Ardisana et al., 2018).

La cantidad de revistas del área de ciencias agrícolas y sus indicadores de calidad pueden estar influenciados por indicadores socioeconómicos, lo que se ha comprobado en otras áreas del conocimiento como las ciencias naturales (Crespo-Gascón, Tortosa y Guerrero-Casado, 2019), comunicación (Rogel-Salazar, Santiago-Bautista y MartínezDomínguez, 2017) y ciencias sociales (Almeida y Grácio, 2017). En el caso particular de las revistas del área de ciencias agrícolas, se han realizado análisis bibliométricos muy específicos, como el realizado a la revista Zootecnia Tropical (Montilla, 2016), el análisis bibliométrico de la revista Centro Agrícola (Paz et al., 2016) y el realizado para la Revista Mexicana de Ciencias Agrícolas. I (Sangerman-Jarquín, Nuñez y NavarroBravo, 2015), así mismo en el trabajo de Guerrero-Casado (2017) se analizaron las 
relaciones entre los indicadores bibliométricos de revistas agropecuarias e indicadores socioeconómicos entre los años 1996 y 2016, tomando como base las revistas Scopus.

Las revistas de ciencias agrícolas en América Latina y en especial las de Suramérica no han sido estudiadas en su conjunto; así mismo, su relación con indicadores socioeconómicos de la región que miden el desarrollo de la agricultura, tampoco se ha analizado, por lo que en la presente investigación se realizó un análisis de los principales indicadores de producción de las revistas de ciencias agrícolas de Suramérica indexadas en SciELO, además de sus relaciones con los indicadores de desarrollo de la agricultura.

\section{Metodología}

\section{Obtención de datos}

Para obtener el número de revistas indexadas en SciELO para el año 2019, se ingresó al portal oficial de la base de datos (https://scielo.org) y se filtró por país y por el área temática de Ciencias Agrícolas. Los indicadores bibliométricos se obtuvieron de la sección Estadísticas de Publicación del portal, filtrando entre los años 2015 y 2020, siendo los mismos los que se describen en la Tabla 1.

Tabla 1. Indicadores bibliométricos de las revistas de ciencias agrícolas indexadas en SCIELO

\begin{tabular}{ll}
\hline Indicador & Descripción \\
\hline Revistas & $\begin{array}{l}\text { Número de revistas indexadas en cada país para } \\
\text { el año } 2019\end{array}$ \\
Documentos Totales & $\begin{array}{l}\text { Número de documentos totales entre 2015 y } \\
2020 \text { por país }\end{array}$ \\
Documentos por Sub-áreas & $\begin{array}{l}\text { Número de documentos por cada sub-área de } \\
\text { publicación de las revistas: Ciencias Agrícolas, } \\
\text { Ingeniería/Sociales y Ciencias Biológicas }\end{array}$ \\
Tipo de Documento & $\begin{array}{l}\text { Número de documentos publicados entre 2015 } \\
\text { y 2O2O por cada tipo: Artículos Originales, } \\
\text { Artículos de Revisión, Informes Breves, Editorial } \\
\text { y Cartas al Editor }\end{array}$ \\
Autores & $\begin{array}{l}\text { Número de autores totales por país entre 2015 } \\
\text { y 2020 }\end{array}$ \\
Procedencia de autores & $\begin{array}{l}\text { Procedencia de los autores de acuerdo dividido } \\
\text { en: Nacionales, Extranjero e Indefinido }\end{array}$ \\
\hline
\end{tabular}

Fuente: elaboración propia

Los indicadores de desarrollo de la agricultura para cada país, se obtuvieron del portal del Banco Mundial ${ }^{1}$ específicamente, los que se describen en la Tabla 2 y los mismos fueron reportados como el promedio entre los años 2015 y 2019. En el caso mundial.org/indicador de Venezuela, país que presentó algunos indicadores sin datos en el periodo de estudio, se tomó el último reportado. 
Tabla 2. Indicadores de desarrollo de agricultura de los países con revistas indexadas en SCiELO

\begin{tabular}{ll}
\hline Indicador & Descripción \\
\hline AG(\%PIB) & Valor agregado de la agricultura como \% del PIB \\
AG(\%C) & Valor agregado de la agricultura como \% del crecimiento anual \\
TAT & Extensión de tierras agrícolas como \% del área de tierra total \\
TACP & $\begin{array}{l}\text { Extensión de tierras destinadas permanentemente al cultivo, como } \\
\text { \% del área de tierra. }\end{array}$ \\
IND & Valor agregado de la industrialización como \% del PIB \\
\hline
\end{tabular}

Fuente: elaboración propia.

Los indicadores bibliométricos se definieron en función al número de revistas de cada país para tener indicadores relativos, es decir, promedios más acordes al objetivo de la investigación (Tabla 3).

Tabla 3. Indicadores bibliométricos relativos en función al número de revistas por país

\begin{tabular}{|c|c|}
\hline Indicador & Descripción \\
\hline DPR & $\begin{array}{l}\text { Documentos publicados en función al número de revistas de cada } \\
\text { país en el periodo } 2015-2020\end{array}$ \\
\hline DCAR & $\begin{array}{l}\text { Documentos publicados en la sub-área de ciencias agrícolas por } \\
\text { revista }\end{array}$ \\
\hline DISR & $\begin{array}{l}\text { Documentos publicados en la sub-área de ingeniería y sociales } \\
\text { por revista }\end{array}$ \\
\hline DCBR & $\begin{array}{l}\text { Documentos publicados en la sub-área de ciencias biológicas por } \\
\text { revista }\end{array}$ \\
\hline AOR & Artículos Originales publicados por Revista \\
\hline ARR & Artículos de Revisión publicadas por Revista \\
\hline IBR & Informes breves publicados por Revista \\
\hline EPR & Editoriales publicados por Revista \\
\hline CER & Cartas al editor publicados por Revista \\
\hline APR & Número de autores por Revista \\
\hline ANR & Número de autores nacionales por Revista \\
\hline AER & Número de autores internacionales por Revista \\
\hline AIR & Número de autores indefinidos por Revista \\
\hline
\end{tabular}




\section{Procesamiento estadístico de los datos}

Para identificar diferencias y similitudes, tanto para los indicadores bibliométricos como para los indicadores de desarrollo de agricultura entre países, se aplicó un análisis de conglomerados basados en valores promedios (Average link age) por metodología Euclidea con datos estandarizados (Cuadras, 2020). Los resultados fueron mostrados mediante dendrogramas.

La relación entre los indicadores bibliométricos y de desarrollo de agricultura se realizó mediante análisis de correlación canónicas, el cual se utiliza para obtener el nivel de correlación entre variables que son independientes. Además, este análisis estadístico permite la obtención de la relación entre variables predictoras métrica y múltiples medidas (Badii et al., 2007), con la finalidad de establecer las posibles asociaciones entre indicadores. Se elaboraron gráficas para describir las tendencias de los indicadores a través de árboles de clasificación. Todos los análisis estadísticos se realizaron con el paquete estadístico InfoStat 2018 y con nivel de significancia $\alpha=0,05$.

\section{Resultados}

Los resultados de los indicadores bibliométricos se observan en la Tabla 4 donde se muestra que el país con el mayor número de revistas de ciencias agrícolas en Suramérica es Brasil con 40 (44,44\%) seguido por Colombia con 19 ( $21,11 \%)$, Argentina y Chile comparten el tercer lugar con 8 revistas cada uno (8,89\%). Durante la investigación se observó que Ecuador y Uruguay no poseen revistas indexadas de ciencias agrícolas en SCIELO, por lo que fueron excluidos del estudio.

Tabla 4. Indicadores bibliométricos de las revistas de ciencias agrícolas de Suramérica indexadas en SciELO.

\begin{tabular}{lcccccccc}
\hline & \multicolumn{7}{c}{ País } \\
\cline { 2 - 8 } Indicador & Argentina & Bolivia & Brasil & Chile & Colombia & Paraguay & Perú & Venezuela \\
\hline Revistas & 8 & 5 & 40 & 8 & 19 & 1 & 4 & 5 \\
DPR & 270,8 & 29,6 & 1023,3 & 491,3 & 212,2 & 165,0 & 120,3 & 132,3 \\
DCAR & 231,0 & 14,8 & 965,2 & 491,3 & 195,5 & 165,0 & 90,3 & 132,6 \\
DISR & 28,1 & 0,0 & 0,0 & 0,0 & 8,5 & 0,0 & 0,0 & 0,0 \\
DCBR & 11,6 & 14,8 & 58,1 & 0,0 & 8,2 & 0,0 & 30,0 & 0,0 \\
AOR & 198,5 & 9,6 & 913,0 & 420,1 & 174,5 & 121,0 & 75,5 & 121,8 \\
ARR & 2,3 & 1,0 & 8,6 & 11,3 & 3,7 & 3,0 & 4,3 & 0,0 \\
IBR & 16,6 & 0,0 & 13,1 & 27,1 & 3,3 & 10,0 & 4,8 & 6,4 \\
EPR & 5,9 & 2,4 & 5,7 & 10,9 & 11,1 & 2,0 & 2,0 & 4,0 \\
CER & 4,0 & 1,8 & 11,8 & 18,4 & 2,4 & 18,0 & 3,0 & 0,0 \\
APR & 275,3 & 16,8 & 1063,7 & 564,9 & 236,8 & 200,0 & 98,3 & 158,6 \\
ANR & 107,9 & 5,2 & 845,7 & 174,9 & 130,3 & 67,0 & 12,8 & 78,6 \\
AER & 16,1 & 3,0 & 85,3 & 279,1 & 58,6 & 37,0 & 17,3 & 22,0 \\
AIR & 151,3 & 8,6 & 132,7 & 110,9 & 48,0 & 96,0 & 68,3 & 58,0 \\
\hline
\end{tabular}


De manera general, Brasil supera al resto de los países de la región en cuanto a los indicadores DPR, DCAR, DCBR, AOR, APR y ANR. El país con el mayor número de documentos publicados en la sub-área de ingeniería y sociales es Argentina y se observa que es la sub-área con menor cantidad de documentos, siendo Colombia el otro país con DISR. La mayor contribución a las revistas en el área fue de los artículos originales (AOR) que representan el $90,2 \%$ de los documentos publicados, con los informes breves en segundo lugar con 3,7\% del total. En relación a la contribución de autores internacionales, las revistas chilenas fueron las que presentaron el mayor índice con un promedio de 279 autores por revista, seguido por las revistas brasileñas con 85 autores internacionales por revista. Las contribuciones nacionales a las revistas estuvieron dominadas por Brasil, Argentina, Chile y Colombia, con Venezuela en el quinto lugar. La mayoría de las revistas en los países presenta una mayor colaboración nacional con relación a la internacional, con la excepción de las revistas de Chile y Perú, así mismo la contribución de autores de nacionalidad indefinida fue mayor a la contribución nacional en las revistas de Argentina, Paraguay y Perú.

Para establecer la distribución de las revistas y sus indicadores bibliométricos en función a los países de Suramérica se realizó el respectivo análisis de conglomerados (Figura 1) donde se observan que los países se agrupan en cinco de ellos.

Figura 1. Dendrodrama de la distribución de conglomerados de los indicadores bibliométricos

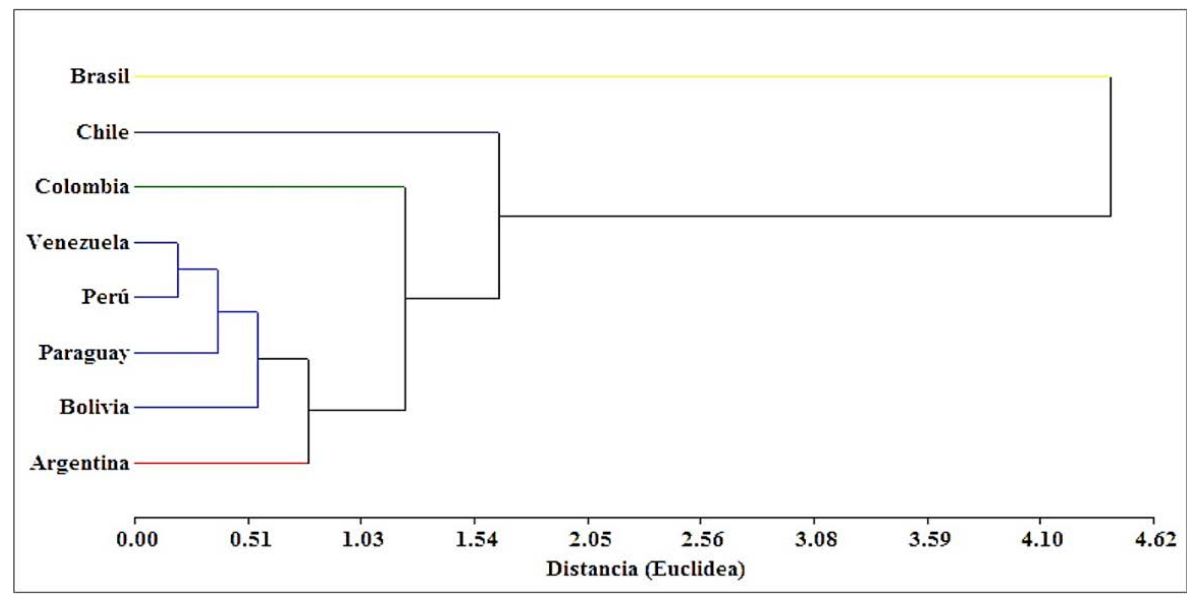

Se observa que Argentina, Brasil, Chile y Colombia se diferencian del resto de los países, los cuales se agrupan en un solo clúster, es decir, a pesar de que tienen diferentes números de revistas de ciencias agrícolas indexadas en SciELO, Bolivia, Paraguay, Perú y Venezuela presentan similitudes significativas en cuanto a los indicadores bibliométricos de las mismas.

Los indicadores de desarrollo de agricultura, se muestran en la Tabla 5. Se observa que el país con el mayor valor agregado en agricultura como \%PIB es Brasil, seguido de Paraguay y Perú.

En cuanto al valor agregado en agricultura de acuerdo al \% de crecimiento, se observa que el mayor valor le corresponde a Bolivia y, por el contrario, Argentina y Venezuela muestran valores negativos de este indicador; una señal de que están pasando por un proceso de retroceso del valor agregado, es decir, no están creciendo en cuanto a la agricultura. 
Tabla 5. Indicadores de desarrollo de agricultura promedios entre 2015 y 2019.

\begin{tabular}{lcccccccc}
\cline { 2 - 8 } & \multicolumn{8}{c}{ País } \\
\hline Indicador & Argentina & Bolivia & Brasil & Chile & Colombia & Paraguay & Perú & Venezuela \\
\hline AG(\%PIB) & 5,8 & 11,1 & 4,6 & 3,8 & 6,3 & 10,2 & 6,9 & 5,0 \\
AG(\%C) & $-2,1$ & 5,7 & 3,4 & 4,0 & 3,7 & 4,7 & 3,2 & $-4,6$ \\
IND & 13,3 & 10,5 & 10,5 & 19,9 & 11.8 & 19,4 & 13,3 & 12,1 \\
TAT & 54,3 & 34,8 & 33,9 & 21,2 & 40,3 & 55,1 & 18,4 & 24,5 \\
TACP & 0,4 & 0,2 & 0,8 & 0,6 & 1,7 & 0,2 & 1,1 & 0,8 \\
\hline
\end{tabular}

Fuente: elaboración propia.

La industrialización muestra valores menos dispersos, con predominio de Chile y Paraguay con 19,9 y 19,4 respectivamente, y menores valores para Bolivia y Brasil. El país con mayor porcentaje de tierras agrícolas en función a su territorio es Paraguay, seguido por Argentina, en tanto Perú posee menos porcentaje de su territorio catalogado como tierras agrícolas. Por otro lado, Colombia presenta el mayor porcentaje de sus tierras agrícolas dedicadas al cultivo permanente, seguido por Perú, Brasil y Venezuela. En tanto Perú posee menos porcentaje de su territorio catalogado como tierras agrícolas.

La agrupación de los países en función a sus indicadores de agricultura se muestra en la Figura 2, en la que se observan cuatro clúster.

Figura 2. Dendrograma de la distribución de conglomerados de los indicadores de desarrollo de agricultura.

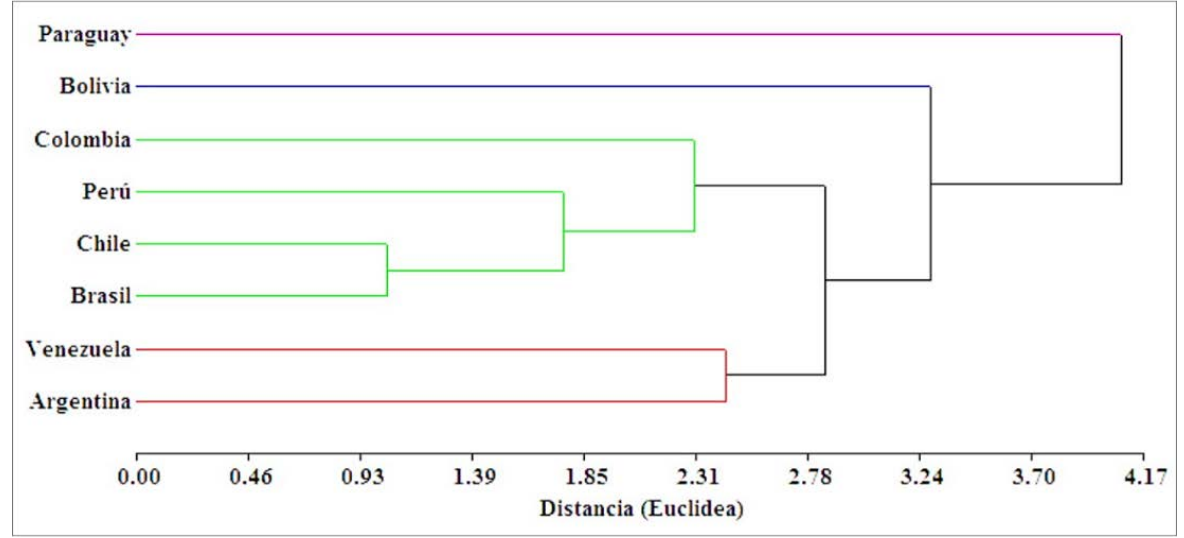

Bolivia y Paraguay no presentan asociación con ningún otro país, por lo que se ubican ambos en clústeres independientes. Argentina y Venezuela ocupan un tercer clúster, lo que indica que sus indicadores son similares estadísticamente y por último el clúster mayor lo componen Brasil, Chile, Colombia y Perú.

Para establecer la relación entre los indicadores bibliométricos con los de desarrollo de agricultura, se escogieron los principales, tales como número de revistas, documentos por revista (DPR), número de autores por revista (APR), contribución internacional (AER); los resultados se muestran en la Tabla 6. Se obtuvo que la industrialización, el porcentaje de tierras agrícolas y el valor agregado de la 
agricultura como \%PIB presentan coeficientes de correlación negativos con todos los indicadores bibliométricos.

Tabla 6. Resultados de coeficientes de correlación canónica entre los indicadores bibliométricos e indicadores de desarrollo de agricultura.

\begin{tabular}{lccccc}
\cline { 2 - 5 } & AG & & & & AG \\
& $(\boldsymbol{\%} \mathbf{C})$ & IND & TAT & TACP & (\%PIB) \\
\hline Revistas & 0,13 & $-0,47$ & $-0,02$ & 0,37 & $-0,45$ \\
DPR & 0,11 & $-0,33$ & $-0,07$ & 0,08 & $-0,58$ \\
APR & 0,11 & $-0,32$ & $-0,07$ & 0,07 & $-0,60$ \\
AER & 0,25 & $-0,27$ & $-0,37$ & 0,03 & $-0,55$ \\
\hline
\end{tabular}

Fuente: elaboración propia

Los coeficientes negativos indican relación inversa entre los indicadores. Los indicadores de industrialización (IND) y el valor agregado en agricultura como \% PIB, son los que mayor relación inversa muestran respecto a los indicadores bibliométricos, siendo el AG (\%PIB) el de mayor importancia ya que su relación es la que muestra los mayores valores de coeficiente de correlación, los cuales indican una relación moderada en los casos de los indicadores documentos publicados, autores totales y autores internacionales y baja respecto al indicador número de revistas (Martínez et al., 2009). La relación positiva mayor se obtuvo entre el número de revistas y las tierras agrícolas destinadas a cultivo permanente, lo que indica una tendencia a que los países con mayor porcentaje de TACP posean mayor cantidad de revistas indexadas en SciELO del área de ciencias agrícolas. La distribución de los países respecto a los dos indicadores con relación positiva $\mathrm{AG}(\% \mathrm{C})$ y TACP, con relación al número de revistas, se muestra en el árbol de clasificación de la Figura 3.

Figura 3. Árbol de clasificación para los indicadores AG (\%C) y TACP respecto al número de revistas por país.

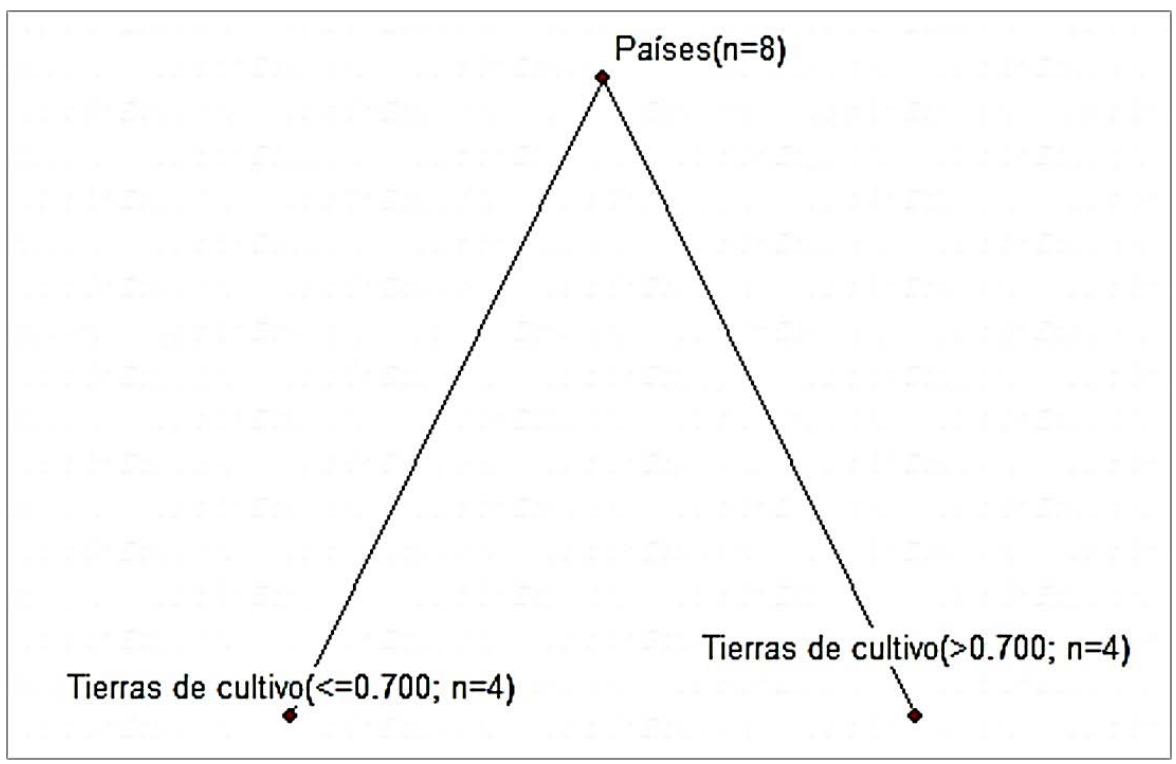


Los resultados del gráfico de árbol de la Figura 3 muestran que existe paridad en la distribución de los países respecto al valor del porcentaje de tierras dedicadas permanentemente al cultivo (TACP). Cuatro países de la región tienen porcentajes de TACP menores a 0,70\% (Argentina, Bolivia, Chile y Paraguay) y cuatro muestran porcentajes de TACP mayores a 0,70\% (Brasil, Colombia, Perú y Venezuela). Esta distribución es consistente con el hecho de que la relación entre los indicadores revistas y TACP es moderada. El árbol de clasificación no muestra al indicador AG (\%C) por ser su relación baja, ya que la contribución a la distribución es mayoritaria del indicador TACP.

Para el caso del indicador DPR, el análisis de correlación canónica mostró que su relación respecto a $\mathrm{AG}(\% \mathrm{C})$ es baja y con $\mathrm{TACP}$ muy baja o despreciable, por lo que el análisis por árbol de clasificación para este indicador bibliométrico (Figura 4) descarta al indicador TACP y solo muestra las relaciones respecto a $A G(\% \mathrm{C})$.

Figura 4. Árbol de clasificación para los indicadores AG (\%C) y TACP respecto al número de publicaciones totales de las revistas por país.

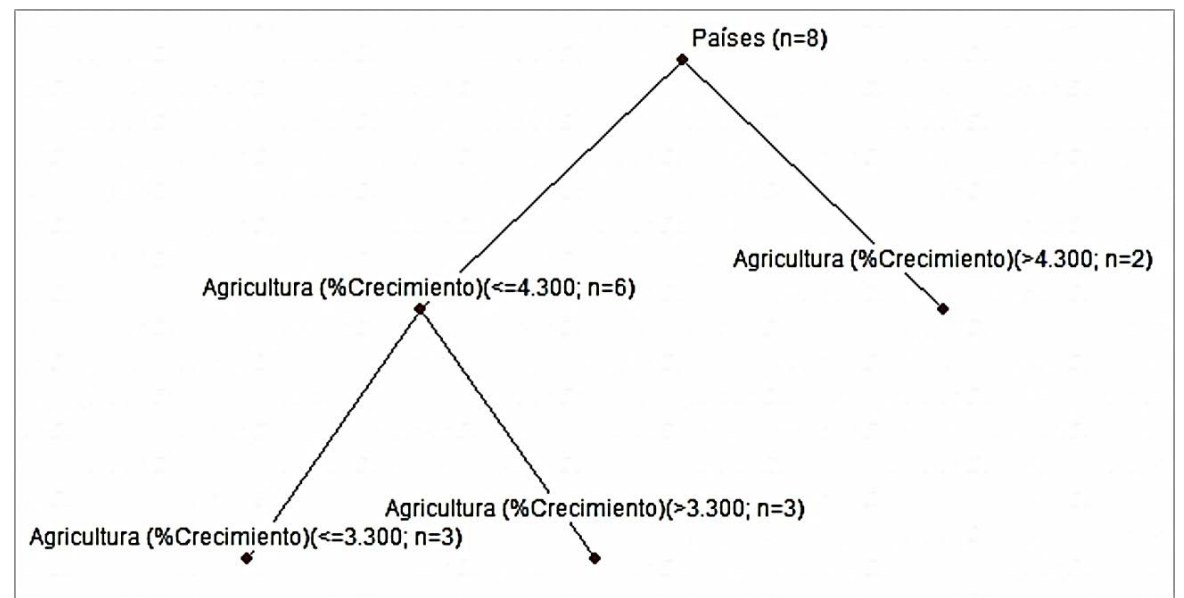

Como se observa en la Figura 4, de los ocho países de la región con revistas de ciencias agrícolas indexadas en SciELO, dos tienen un AG (\%C) mayor a 4,3\%, los cuales son Bolivia con el DPR más bajo de todos y Paraguay, que tiene un DPR intermedio, que a su vez supera a otros países. Entre los países con menor porcentaje de AG $(\% \mathrm{C})$ existe paridad para un valor referencial de 3,3\%, clasificándose tres de ellos por debajo (Argentina, Perú y Venezuela) y tres por encima (Brasil, Chile y Colombia). Destacan los valores negativos de AG (\%C) de Argentina y Venezuela, lo que indica que estos dos países no han experimentado crecimiento en el valor agregado de la agricultura, por el contrario, han mostrado un retroceso en este aspecto.

La distribución de los países en función a la relación entre el indicador bibliométrico AER y los indicadores AG (\%C) y TACP se muestra en la Figura 5, en la que se observa que solo el indicador AG $(\% \mathrm{C})$ se relaciona con $\mathrm{AER}$, lo que coincide con el resultado de la correlación canónica, donde se observa una relación mayor para AG (\%C) en relación con TACP. 
Figura 5. Árbol de clasificación para los indicadores AG (\%C) y TACP respecto al número de autores internacionales en las revistas por país.

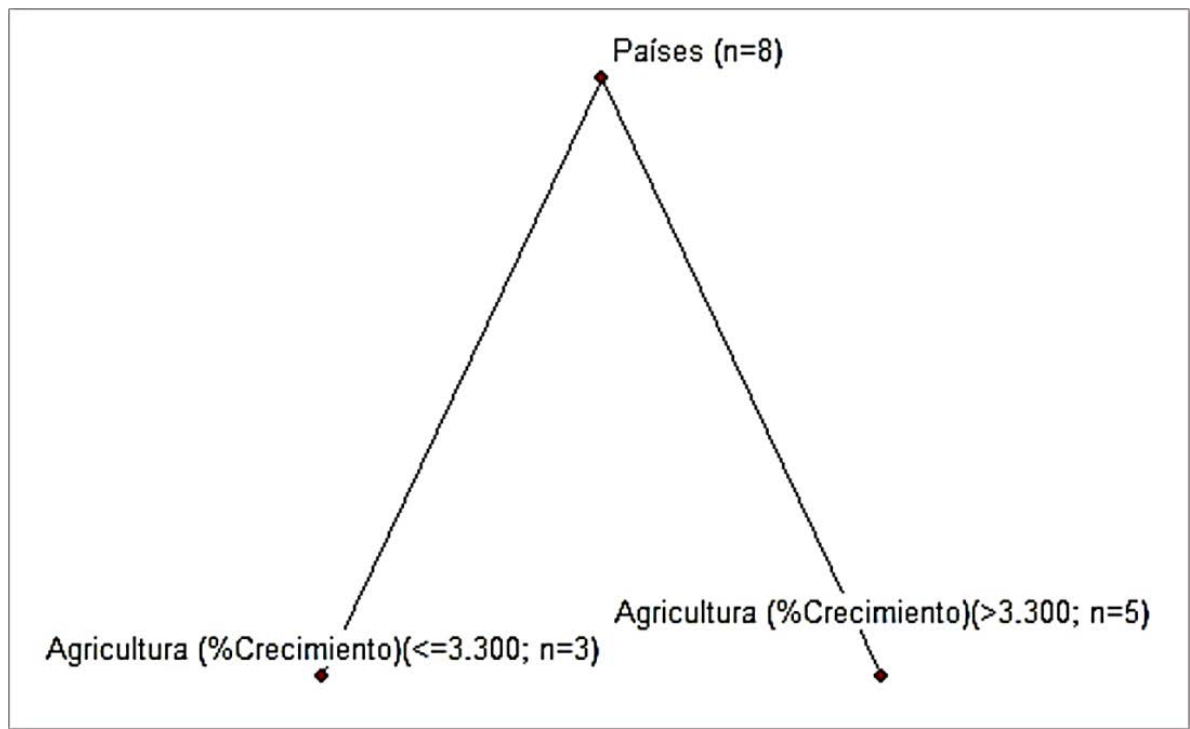

En la Figura 5 se observa que tres de los países con valores más bajos de contribución internacional a las revistas, presentan bajos valores de AG (\%C), es decir menores a $3,3 \%$ de crecimiento, ellos son Argentina, Perú y Venezuela. Los cinco países restantes, superan el 3,3\% de AG (\%C), entre ellos los de mayor cantidad de AER (Brasil, Chile, Colombia y Paraguay), sin embargo en este grupo también se encuentra Bolivia que presenta el menor valor de AER, lo que incide en la relación baja observada.

El análisis de las relaciones de los indicadores bibliométricos con los indicadores de agricultura y desarrollo cuya influencia fue negativa, según la correlación canónica, se inicia con el número de revistas por país (Figura 6).

Figura 6. Árbol de clasificación para los indicadores IND, AG (\%PIB) y TAT respecto al número de revistas por país.

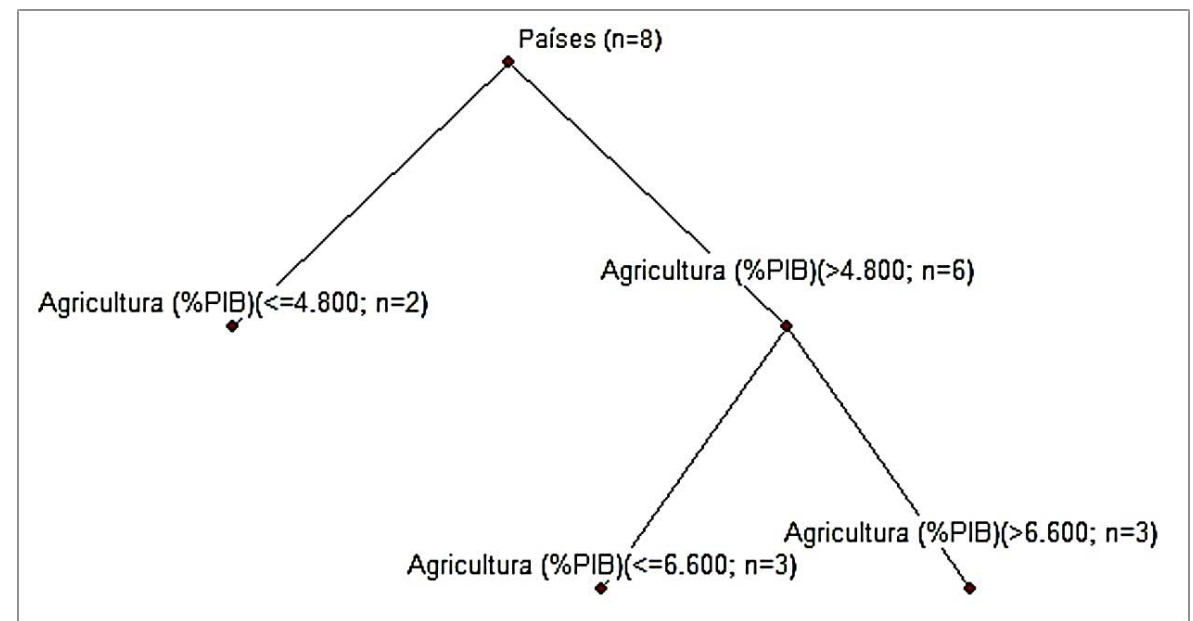


Se observa que en el árbol de clasificación se tomó en cuenta solo el indicador AG (\%PIB), lo que indica que respecto al número de revistas, este es el indicador de mayor relevancia estadística. Dos países mostraron valores de AG (\%PIB) menor a $4,8 \%$ (Brasil y Chile), estos países junto al que ocupa el tercer lugar tienen el mayor número de revistas, esto indica la relación de proporcionalidad inversa encontrada con la correlación canónica. Los seis países restantes muestran paridad respecto al valor de referencia 6,6\%. Argentina, Colombia y Venezuela presentan valores de AG (\%PIB) menores al valor referencial, aun cuando cuentan con 8, 19 y 5 revistas que son cantidades importantes respecto al total. Bolivia, Paraguay y Perú superan el valor referencial como los países con mayores AG (\%PIB), sin embargo presentan pocas revistas y en el caso de Paraguay solo tiene una, lo que también refuerza la poca relación directa entre los indicadores analizados.

El número de documentos por revista (DPR) mostró la distribución respecto a los indicadores de influencia negativa como se observa en la Figura 7. Es importante resaltar que el análisis por árbol de clasificación indicó que el indicador IND no tiene una contribución importante y por eso se descartó del análisis y el paquete estadístico generó el árbol con los indicadores AG (\%PIB) y TAT.

Figura 7. Árbol de clasificación para los indicadores IND, AG (\%PIB) y TAT respecto al número de documentos publicados por revistas en cada país.

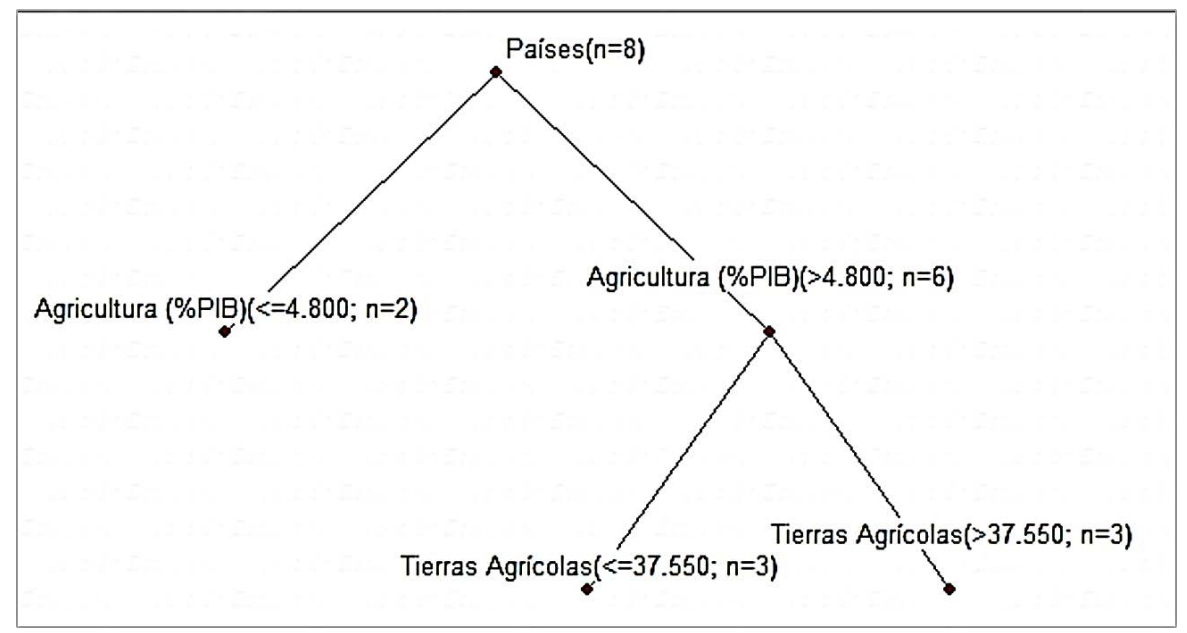

Dos de los países (Brasil y Chile) presentan valores de AG (\%PIB) menores a 4,8\%, siendo también dos de los que poseen menores porcentajes de TAT, pero los cantidades de DPR mayores. De los seis restantes, tres tienen porcentajes de tierras agrícolas mayores a 37,55\% (Argentina, Colombia y Paraguay) y sus revistas tienen cantidades de documentos importantes y tres con menos de $37,55 \%$ de TAT (Bolivia, Perú y Venezuela), siendo también los tres países con menores cantidades de DPR. Para el caso del indicador de número de autores por revistas (APR), el comportamiento fue el mismo que para DPR, lo que está acorde con la similitud encontrada entre los coeficientes de correlación canónica de estos dos indicadores.

El indicador AER también mostró relación, según el análisis de árbol de clasificación, con AG (\%PIB) y con TAT, como se observa en la Figura 8, donde Chile es el país que presenta el menor porcentaje de AG (\%PIB) y que el mismo es menor a 4,2\%, a pesar de que es el que presenta la mayor contribución de autores internacionales con 279,10 por revista. 
Figura 8. Árbol de clasificación para los indicadores IND, AG (\%PIB) y TAT respecto al número de autores internacionales por revistas en cada país.

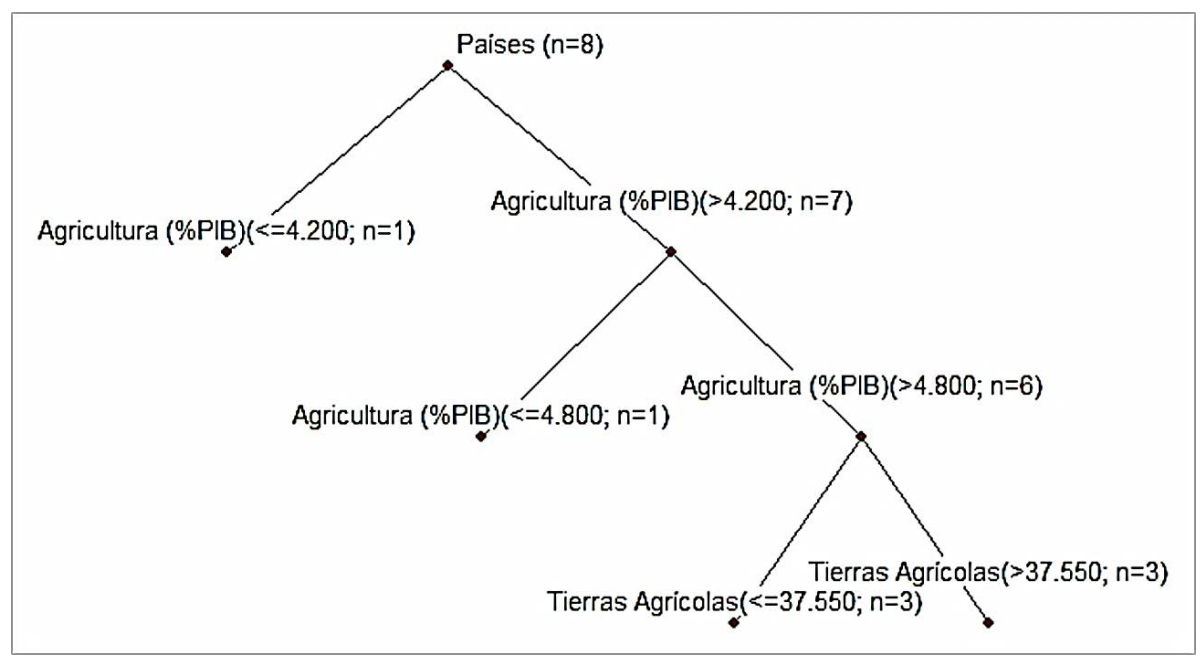

Brasil se ubica solo como el país con AG (\%PIB) entre 4,2 y 4,8\%, que lo deja como el segundo país con el menor valor de este indicador a pesar de ser el segundo en contribución extranjera a sus revistas de ciencias agrícolas. Entre los países con AG (\%PIB) mayores a 4,8\%, Argentina, Colombia y Paraguay superan el $37,55 \%$ de su superficie disponible para agricultura, aun cuando Argentina tiene baja contribución en AER. El resto de los países, Bolivia, Perú y Venezuela dedican menos de 37,55\% de su territorio para la agricultura, aunque en relación a su AER, Perú y Venezuela superan a Argentina.

\section{Discusión}

En Suramérica para el año 2019 se contabilizaron 90 revistas del área de ciencias agrícolas indexadas en SCiELO, lo que corresponde al 10,61\% del total de 848 revistas activas para el mismo año en los países de muestra. Este porcentaje de revistas es bajo, si se considera que la actividad agrícola es fundamental para el desarrollo de la subregión. Se corroboró que los países con mayor cantidad relativa de revistas de ciencias agrícolas en relación con la cantidad de revistas que tienen indexadas en SciELO, son Bolivia (17,86\%), Brasil y Venezuela (13,51\%) y Perú $(11,76 \%)$. Lo anterior muestra que aunque Brasil y Colombia representan los países con mayor porcentaje de revistas de ciencias agrícolas, en relación al total de estas (44,44 y $21,11 \%$ respectivamente), respecto al total de revistas indexadas por país, otros como Bolivia, Perú y Venezuela muestran una mayor tendencia interna hacia la difusión e indexación de sus revistas del área, lo que indica la importancia que para estos países representa esta área del conocimiento y la difusión de la investigación, pues como lo indican Ganga, Paredes y Pedraja-Rejas (2015) las publicaciones científicas proporcionan acceso a nuevos saberes, por lo que son el vehículo efectivo y riguroso para dar a conocer el conocimiento que se está produciendo en la disciplina.

El análisis de conglomerados realizado con los indicadores bibliométricos, mostró que cuatro países de la región se destacan por encima del resto y que se ubican en clústeres diferentes, estos países son además los de mayor producción científica general, según el portal de Scimago Journal er Rank (Argentina, Brasil, Chile y Colombia) lo que demuestra que no solo dominan las métricas científicas globales, sino también las del 
área temática estudiada. Esta tendencia también fue reportada para otras áreas temáticas como las ciencias de la salud, donde Argentina, Brasil, Chile y Colombia dominan las publicaciones científicas en Suramérica y a nivel latinoamericano también destaca en esta área Cuba (Carvajal-Tapia y Carvajal-Rodríguez, 2019). El resto de los países con revistas de ciencias agrícolas indexadas en SCiELO se agruparon en un solo clúster, lo que indica similitud estadística de sus indicadores bibliométricos, a pesar de las diferencias en cuanto al número de revistas y en la cantidad de documentos publicados, por lo que, así como lo manifiestan Cepeda, Pazmiño y Medrano (2018) aun cuando la producción científica en Latinoamérica ha ido en aumento, todavía es poco en relación con el resto de los países del mundo; solo Brasil se destaca por su producción.

En relación con las revistas del área de ciencias agropecuarias en Latinoamérica, Guerrero-Casado (2017) también observó que, aunque el número de revistas indexadas en Scopus ha ido en aumento, aun se observan grandes diferencias, sobre todo en cuanto a los documentos publicados a pesar de la importancia de esta área de conocimiento para el desarrollo de los países. De igual manera, otros autores al analizar revistas específicas del área destacan que, a pesar de la importancia de las investigaciones para el desarrollo de los países, el número y calidad de las publicaciones debe ser revisado y analizado bajo la perspectiva del desarrollo de la región (Sangerman-Jarquín, Nuñez y Navarro-Bravo, 2015; Montilla, 2016; Paz, Peralta y Hernández, 2016). La agricultura y la agroindustria se han visualizado como actividad prioritaria a nivel mundial y América Latina posee un enorme potencial para promover el desarrollo de los países de la región (Barrantes et al., 2013), destacándose Suramérica por ser fuente de origen de muchos cultivos; sin embargo, para ello deben ocurrir cambios importantes en la gobernanza, que permitan no solo el desarrollo de tecnologías y de procesos mejorados, sino, además, en la difusión del conocimiento y la investigación.

El análisis de los indicadores de desarrollo de agricultura mostró que los países se agrupan en cuatro conglomerados o clústeres, con Bolivia y Paraguay destacándose del resto, sobre todo por su valor agregado de agricultura en \%PIB, la industrialización de la misma y el porcentaje de su territorio disponible como tierras agrícolas. En el caso de Paraguay, es un país cuya economía está basada en el agro y en la última década se ha caracterizado por un marcado dinamismo del sector agrícola, impulsado por el incremento de productividad y competitividad de las principales cadenas productivas (Ferreira et al., 2015), lo que también es resaltado por la Organización de las Naciones Unidas para la Alimentación y la Agricultura (FAO, 2017). Bolivia se presenta como el país con el mayor \%PIB como valor agregado de agricultura y con el mayor \%Crecimiento en el sector, así como el tercero con mayor porcentaje de territorio con tierras agrícolas, lo que también lo destaca de los demás países, esto a pesar de los controles sobre la agricultura que se aplicaron en los últimos tiempos en el país (Vera, 2012).

Al relacionar los indicadores bibliométricos con los socioeconómicos, es claro que no existe una asociación considerablemente fuerte entre ellos, con un predominio de dependencia de los indicadores de industrialización y valor agregado de la agricultura (\%PIB), en ambos casos de forma negativa, es decir, una asociación inversa. Pareciera entonces que los países con mayor industrialización y \%PIB como valor agregado de agricultura, son los que muestran menores valores de los indicadores bibliométricos de sus revistas de ciencias agrícolas. La asociación positiva mayor se observó para el número de revistas con el indicador de tierras agrícolas dedicadas al cultivo permanente. La observación anterior coincide con lo concluido por GuerreroCasado (2017) quien al analizar las publicaciones agropecuarias de América Latina menciona relaciones de mediana importancia y además reporta que los países que más dependen de la agricultura y la ganadería debido a aportación al PIB y al porcentaje de población rural, presentan menor cantidad de publicaciones científicas en Scopus. 
El bajo número de publicaciones y de revistas disponibles en la región es un factor que puede frenar el desarrollo del sector productivo, sobre todo en los países donde las actividades agropecuarias son parte importante de la economía. De igual manera, es necesario destacar que las investigaciones científicas y la divulgación de estas en revistas de especialidad, generan un impulso significativo al desarrollo de las sociedades, lo que hace que los países menos desarrollados deban invertir más en sus investigaciones y, sobre todo, en la promoción de sus revistas ya que la producción científica se ve influenciada por la inversión en IED (Ganga, Paredes y Pedraja-Rejas, 2015). Aun cuando se ha evidenciado en otras investigaciones que ha existido un incremento en la producción científica en el área agropecuaria, la misma no está en sintonía con la inversión de los países y con sus indicadores de desarrollo (Montilla, 2016); por otro lado, en algunos casos se han presentado también disminución en la producción científica, lo que repercute negativamente en los indicadores de visibilidad de las revistas (Ortega et al., 2015).

En cuanto a las asociaciones positivas entre los indicadores, además de la señalada entre número de revistas y tierras agrícolas de cultivos permanentes, la segunda mejor asociación fue observada entre el número total de autores internacionales y el \% de crecimiento de la agricultura, lo que indica una tendencia importante de aporte internacional a las publicaciones y, por ende, al crecimiento del valor agregado de la agricultura. A respecto, Paz, Peralta y Hernández (2016) destacan la importancia de la contribución externa a las revistas, para evitar la dañina endogamia editorial, que evidentemente puede limitar la indexación y visibilidad de las revistas en bases de datos importantes.

\section{Conclusiones}

En Suramérica, en relación con las revistas del área de ciencias agrícolas indexadas en SciELO, destacan por sus indicadores bibliométricos Argentina, Brasil, Chile y Colombia, los cuales se muestran como los países con los mejores indicadores y además muestran diferencias significativas entre ellos, donde se destaca Brasil por su mayor número de revistas y su mayor producción científica. El resto de los países de la región, a pesar de contar con números diferentes de revistas indexadas, no mostraron diferencias al analizarse sus indicadores bibliométricos en conjunto, por lo que se ubican en un conglomerado o clúster único.

Las relaciones entre los indicadores bibliométricos y los de desarrollo de agricultura mostraron asociaciones de muy bajas a moderadas, siendo las mayores las asociaciones negativas respecto al nivel de industrialización y al valor agregado de la agricultura como $\%$ PIB, que evidencia que estos dos indicadores están relacionados de forma moderada e inversa con los indicadores bibliométricos estudiados para las revistas de ciencias agrícolas de Suramérica indexadas en SciELO. Las relaciones positivas mayores fueron entre el número de revistas y el porcentaje de las tierras agrícolas destinadas el cultivo permanente, y entre el número de autores internacionales y el valor agregado de la agricultura como porcentaje del crecimiento.

Una mayor cantidad de revistas y publicaciones en el área de ciencias agrícolas no parece estar asociada a mayores resultados de indicadores agrícolas, lo que evidencia un desequilibrio en la importancia de las actividades agropecuarias para la región y la producción científica. 


\section{Referencias bibliográficas}

》 Almeida, Cátia Candida y Maria Cláudia Cabrini Grácio. 2017. Factor de impacto de revistas de la América Latina en ciencia social: Un estudio comparativo entre las bases Scopus y Web of Science. En Revista Guillermo de Ockham. Vol. 15, no. 2, 69-74. <http://dx.doi.org/10.21500/22563202.3052>

» Ardisana, Eduardo, Bárbara Gaínza, Antonio Torres y Osvaldo Fosado. 2018. Agricultura en Sudamérica: la huella ecológica y el futuro de la producción agrícola. En Revista Chakiñan de Ciencias Sociales y Humanidades. No. 5, 90-101. <http://scielo.senescyt.gob.ec/pdf/rchakin/n5/2550-6722-rchakin-05-00090. pdf> [Consulta: 02 junio 2020].

» Badii, M.H., J. Castillo, K. Cortez, A. Wong y P. Villalpando. 2007. Análisis de correlación canónica (ACC) e investigación científica. En Innovaciones de $\mathrm{Ne}$ gocios. Vol. 4, no. 2, 405-422. <http://eprints.uanl.mx/12486/1/A9\%20\%281\%29. pdf> [Consulta: 23 mayo 2020].

"Barrantes, Roxana, Julio Berdegué, Alain de Janvry, Eugenio Díaz-Bonilla, Desirée Elizondo, Gustavo Gordillo, Ana María Ibáñez, Roberto Junguito, Reed Hertford, Carlos Pomareda, Alberto Valdés, Juan Manuel Villasuso y Antonio Yúnez-Naude. 2013. Agricultura y desarrollo en América Latina: gobernanza y políticas públicas. Buenos Aires: Editorial Teseo.

»Carvajal-Tapia, Aarón y Eduardo Carvajal-Rodríguez. 2019. Producción científica en ciencias de la salud en los países de América Latina, 2006-2015: análisis a partir de SciELO. En Revista Interamericana de Bibliotecología. Vol. 42, no. 1, 15-21. <http://doi.org/10.17533/udea.rib.v42n1ao2>

" Cepeda, Katuska, Lucila Pazmiño y Eva Medrano. 2018. Evolución de la Investigación Científica en América Latina. En RECIMUNDO Revista Científica Mundo de la Investigación y el Conocimiento. Vol. 2, no. 2, 464-476. <http://doi. org/10.26820/recimundo/2.(2).2018.464-476>

"Crespo-Gascón, Sofía, Francisco Tortosa y José Guerrero-Casado. 2019. Producción de revistas científicas en América Latina y El Caribe en Scopus, Journal Citation Reports y Latindex en el área de los recursos naturales: su relación con variables económicas, ambientales y de inversión en investigación. En Revista española de Documentación Científica. Vol. 42, no. 1, e224. < https://doi.org/10.3989/redc.2019.1.1533>

»Cuadras, Carles. 2020. Nuevos métodos de análisis multivariante. Barcelona: CMC Editions.

»Escobar, Germán. 2016. La relevancia de la agricultura en América Latina y el Caribe. Buenos Aires: Fundación Friedrich Ebert (FES).

»Ferreira, Manuel, Fabricio Vázquez, Valeria Gutiérrez, Nidia Corvalán y José Dacak. 2015. Agricultura y desarrollo en Paraguay. Asunción: investor.

» Ganga, Francisco, Lorena Paredes y Liliana Pedraja-Rejas. 2015. Importancia de las publicaciones académicas: algunos problemas y recomendaciones a tener en cuenta. En Idesia (Arica). Vol. 33, no. 4, 111-119. <http://dx.doi.org/10.4067/ So718-34292015000400014>

»Gliessman, Stephen. 2013. Agroecología: Plantando las raíces de la resistencia. En Agroecología. Vol. 8, no. 2, 19-26. <https://revistas.um.es/agroecologia/article/view/212151/168371> [Consulta: 02 junio 2020]. 
》Guerrero-Casado, José. 2017. Producción científica latinoamericana indexada en Scopus en el área de las ciencias agropecuarias: análisis del período 19962016. En IDESIA (Chile). Vol. 35, no. 4, 27-33. <https://scielo.conicyt.cl/pdf/idesia/v35n4/0718-3429-idesia-35-04-00027.pdf> [Consulta: 15 junio 2020].

"Martínez, Rosa, Leonel Tuya, Alberto Pérez y Ana Cánovas. 2009. El coeficiente de correlación de los rangos de Spearman caracterización. En Revista Habanera de Ciencias Médicas. Vol. 8, no.2, 1-19. <http://scielo.sld.cu/pdf/rhcm/v8n2/ rhcm17209.pdf> [Consulta: 23 mayo 2020].

»Mazzaro, Cecilia. 2010. Comunicar la ciencia. Perspectivas, problemas y propuestas psiencia. En Revista Latinoamericana de Ciencia Psicológica. Vol. 2, no. 2, 122-127. <https://www.redalyc.org/pdf/3331/333127104010.pdf> [Consulta: 16 mayo 2020].

»Montilla, Leomar. 2016. Análisis de la producción científica de los artículos de la Revista Zootecnia Tropical del Instituto Nacional de Investigaciones Agrícolas (2006-2013). En Biblios. No. 65, 1-14. <https://dx.doi.org/10.5195/biblios.2016.315>

»Organización de las Naciones Unidas para la Alimentación y la Agricultura. 2017. Análisis de capacidades técnicas e institucionales Paraguay. Santiago: FAO.

»Ortega, Elianes, Yamila Díaz, Francisco Soto y René Florido. 2015. Productividad y visibilidad de la revista "cultivos tropicales" 2009-2013. En Cultivos Tropicales. Vol. 36, no. 3, 20-26.

»Paz, Luis, María Peralta y Eduardo Hernández. 2016. Estudio bibliométrico de la Revista Centro Agrícola, Cuba. En E-Ciencias de la Información. Vol. 6, no. 2, 1-23. <http://dx.doi.org/10.15517/eci.v6i2.2573>

» Rodríguez, Luis. 2010. Origen y evolución de la papa cultivada. Una revisión. En Agronomía Colombiana. Vol. 28, no. 1, 9-17. <http://www.scielo.org.co/pdf/agc/ v28n1/v28n1ao2.pdf> [Consulta: 30 mayo 2020].

» Rogel-Salazar, Rosario, Irvin Santiago-Bautista y Néstor Martínez-Domínguez. 2017. Revistas científicas latinoamericanas de Comunicación indizadas en WoS, Scopus y bases de datos de Acceso Abierto. En Comunicación y Sociedad. Vol. 30, 167-196. <http://www.scielo.org.mx/pdf/comso/n30/o188-252X-comso-30-00167.pdf> [Consulta: 30 mayo 2020].

"Salaverry, Oswaldo. 2012. la comida en el antiguo Perú: Haku mikumusum (jvamos a comer!). En Rev Peru Med Exp Salud Pública. Vol. 29, no. 3, 409-413. <http://www. scielo.org.pe/pdf/rins/v29n3/azov29n3.pdfs [Consulta: 27 mayo 2020].

» Sánchez-Tarragó, Nancy, Alejandro Caballero-Rivero, Piotr Trzesniak, Dania Deroy, Raimundo Dos Santos y Juan-Carlos Fernández-Molina. 2016. Las revistas científicas en América Latina hacia el camino del acceso abierto: un diagnóstico de políticas y estrategias editoriales. En TransInformação, Campinas. Vol. 28, no. 2, 159-172. <http://dx.doi.org/10.1590/2318-08892016000200003>

» Sangerman-Jarquín, Dora, Juan Núñez y Agustín Navarro-Bravo. 2015. Análisis de la colaboración científica en artículos publicados por la Revista Mexicana de Ciencias Agrícolas. I. En Revista Mexicana de Ciencias Agrícolas. Vol.6, no. 8, 1867-1877. <https://cienciasagricolas.inifap.gob.mx/index.php/publicaciones> [Consulta: 15 mayo 2020].

"Vera, Diego. 2012. Agricultura y empleo rural agropecuario en Bolivia. Consecuencias del control de precios en el sector agropecuario. En LAJED. No 17, 113-134. <http://www.scielo.org.bo/pdf/rlde/n17/n17_a05.pdf> [Consulta: 18 junio 2020]. 